\title{
Enhancing PUFA-rich polar lipids in Tisochrysis lutea using adaptive laboratory evolution (ALE) with oscillating thermal stress
}

\author{
Gachelin Manon 1, * , Boutoute Marc ${ }^{1}$, Carrier Gregory 2, Talec Amélie ${ }^{1}$, Pruvost Eric ${ }^{1}$, \\ Guihéneuf Freddy ${ }^{1,3}$, Bernard Olivier ${ }^{4}$, Sciandra Antoine ${ }^{1}$
}

${ }^{1}$ Laboratoire d'Océanographie de Villefranche (LOV, UMR 7093), Sorbonne Universités, CNRS, Station zoologique, 181 Chemin du Lazaret, 06230, Villefranche-sur-Mer, France

2 Laboratoire Physiologie et Biotechnologie des Algues (PBA), IFREMER, Nantes, France

3 SAS Inalve, 181 chemin du lazaret, 06230, Villefranche-sur-Mer, France

${ }^{4}$ Biocore, INRIA Sophia Antipolis Méditerranée, Valbonne, France

* Corresponding author : Manon Gachelin, email address : manon.gachelin@obs-vlfr.fr

\begin{abstract}
:
Adaptive laboratory evolution is a powerful tool for microorganism improvement likely to produce enhanced microalgae better tailored to their industrial uses. In this work, 12 wild-type strains of Tisochrysis lutea were co-cultivated under increasing thermal stress for 6 months. Indeed, temperature was oscillating daily between a high and a low temperature, with increasing amplitude along the experiment. The goal was to enhance the polyunsaturated fatty acid content of the polar lipids. Samples were taken throughout the evolution experiment and cultivated in standardized conditions to analyze the evolution of the lipid profile. Genomic analysis of the final population shows that two strains survived. The lipid content doubled, impacting all lipid classes. The fatty acid analyses show a decrease in SFAs correlated with an increase in monounsaturated fatty acids (MUFAs), while changes in polyunsaturated fatty acid (PUFAs) vary between both photobioreactors. Hence, the proportion of C18-MUFAs (18:1 n-9) and most C18-PUFAs (18:2 n-6, 18:3n-3, and 18:4 n-3) increased, suggesting their potential role in adjusting membrane fluidity to temperature shifts. Of particular interest, DHA in polar lipids tripled in the final population while the growth rate was not affected
\end{abstract}

\section{Key points}

Adaptive laboratory evolution on a mix of $12 \mathrm{~T}$. lutea strains led to survival of $2-$ Thermal stress impacted cell size, total lipid cell content, and all lipid classes DHA cell content partitioned to polar lipids tripled throughout the experiment 
Keywords : Tisochrysis lutea, Polar lipids, Adaptive laboratory evolution (ALE), Temperature, DHA 


\section{Introduction}

Microalgae are known for their diverse and original metabolisms, leading to an ability to produce many bioactive compounds depending on species and cultivation strategies. They therefore offer potential applications in food and feed, cosmetics, and pharmaceutics (Spolaore et al. 2006). Microalgae have an interesting potential for food and feed, as natural resources become more and more limited (FAO 2017). The phenotypic plasticity of their metabolisms allows to cope with environmental fluctuations. It can also be enhanced to better fit their cultivation conditions and optimize their industrial productivities. Adaptive laboratory evolution (ALE) is widely used for strain improvement of yeast and bacteria (Dragosits and Mattanovich 2013; Wang et al. 2014). Few studies have been published, which tested evolution protocols on microalgae, but they obtained promising results to enhance different metabolic pathways. Bonnefond et al. (2017) enhanced lipid productivity. Fu et al. (2012, 2013) increased carotenoid content. Perrineau et al. (2013) observed higher tolerance to high salinity. Li et al. (2015) obtained cells resistant to high $\mathrm{CO} 2$ concentration. Wang et al. (2016) enhanced the phenol degradation capacity.

Fish oils are often the lipid sources in feed, resulting in pressuring wild fish stocks. Moreover, fish oil supply is unsecured, with fluctuating quality and quantity along the year. There is therefore room for another source of lipids (Naylor et al. 2009). Some microalgae species can accumulate 20 to $50 \%$ lipid in dry biomass and even up to $80 \%$ under certain conditions (Santos-Sanchez et al. 2016; Bellou et al. 2014). Their fatty acid (FA) profiles can be rich in specific essential fatty acids, $\omega 3$-, and $\omega 6$-long-chain polyunsaturated fatty acids (LC-PUFAs) (over $20 \%$ of total fatty acids). Temperature is a factor that strongly influence growth rate and lipid content (Roleda et al. 2013; Zhu et al. 2016) with strong species-specific effects (Zhu et al. 2016; Renaud et al. 1995; Thompson et al. 1992; Renaud et al. 2002; Bonnefond et al. 2017). Temperature also affects the saturated/unsaturated FA ratio and profile 
(Renaud et al. 1995; Thompson et al. 1992). It has been hypothesized that this acclimation contributes to maintain membrane fluidity (Ackman et al. 1968; Mortensen et al. 1998).

Temperature has already been used as a selection factor in long-term adaptive experiments to improve lipid productivity. A 6-month experiments with Tisochrysis lutea consisting in applying daily fluctuating temperatures of increasing amplitude (Bonnefond et al. 2017) contributed to enlarge thermal niche $(+16 \%)$ together with the maximal growth rate $(+9 \%)$. It also resulted in modifying the FA profile. The key idea of this evolution experiment was to maintain a dynamic selection pressure to force the cells to adapt and therefore survive to their environment.

Here, we use ALE for enhancing Tisochrysis lutea in PUFA-rich polar lipids. This oleaginous microalga is commonly used in aquaculture (Rico-Villa et al. 2006) especially for its significant content of docosahexaenoic acid (DHA) (Renaud et al. 1995; Thompson et al. 1992; Liu et al. 2013). Twelve wild-type strains were equiproportionally mixed in the inoculumto increase initial genomic diversity. This mix of strains was continuously cultivated for 6 months under thermal stress. The ALE experiment consisted in daily fluctuating temperature with increasing amplitude. The stress intensity (amplitude of the fluctuations) was increased weekly, balancing between a reasonable growth rate and the induction of durable modifications in the fatty acid profile of polar lipids. Genomics analyses were performed at the end of each selection cycle to determine the remaining strains and track genomic modifications.

\section{Material and methods}

\section{Microalgal strains}

The 12 strains of the photoautotrophic microalga $\mathrm{Ti}$ sochrysis lutea used in this study (supplementary data, table S1) originated from culture collections (CCAP927/14, CCMP463, RCC179, RCC1344, RCC3691, RCC3692, RCC3693, RCC3699, NIVA 4-91, IMFG, and Argenton 1998). The strain Tiso S-5 (maintained at PBA laboratory, IFREMER, Nantes, France) was derived from the original strain CCAP927/14 after a sequential selection procedure ( $1 \%$ lowest lipid content).

\section{Cultivation device and protocol}

\section{The adaptive laboratory evolution device}

The microalgae were grown under computer-controlled conditions in duplicate flat panel photobioreactors (TL1 and TL2), $6 \mathrm{~cm}$ culture thickness. These home-designed water-jacketed 1.8 L photobioreactors (called "selectiostats," see Fig. 1) were specially designed to maintain and survey continuous cultures for several months. Biofouling was prevented as much as possible by bubbling $0.2 \mu \mathrm{m}$ filtered air along the transparent walls of the selectiostats, and their simple geometry makes the optical measurements of PAR and turbidity reliable.

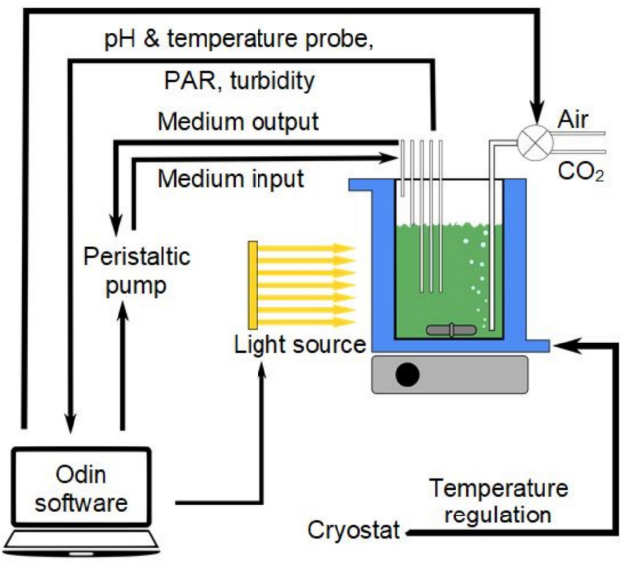

Fig. 1 Simplified diagram of a selectiostat and its controlled parameters

Cultures were grown in turbidostat mode, i.e., dilution rate was automatically regulated to maintain the turbidity and thus cell density between 3.5 and 4 million cells $/ \mathrm{mL}$. Turbidity was estimated from the beam attenuation of a light source at $750 \mathrm{~nm}$ through the thickness of the culture. It was kept sufficiently low to avoid self-shading and nutrient limitation. Continuous light was provided by a LED panel (Nichia NVSL219BT $2700^{\circ} \mathrm{K}$ ) placed on one side of the photobioreactor. Intensity of incident light in the visible spectrum (PAR; photosynthetically active radiation) was set at $300 \mu \mathrm{mol}$ photons $/ \mathrm{m} 2 / \mathrm{s}$, and occasionally controlled with a spherical Biosphere Instrument QSL-100 probe. PAR was also continuously measured with a flat Skye PAR Quantum Sensor probe positioned against the transparent wall on the other side of the photobioreactor. Measured PAR behind the photobioreactor was oscillating between 150 and $200 \mu \mathrm{mol}$ photons $/ \mathrm{m} 2 / \mathrm{s}$. Cultures were homogenized through magnetic stirring and bubbling. $\mathrm{pH}$ was continuously measured and regulated at 8.2 by controlled micro-injections of $\mathrm{CO} 2$ in the bubbled air. The renewing medium was prepared in 20-L Nalgen tanks with autoclaved $\left(121^{\circ} \mathrm{C}, 30 \mathrm{~min}\right), 0.2-\mu \mathrm{m}$ filtered natural seawater, enriched with sterile Conway-Walne medium (Walne 1974). Medium was introduced in the selectiostats with a peristaltic pump (Gilson Minipuls 3), through a $0.22-\mu \mathrm{m}$ Whatman filter. The flow-rate of the peristaltic pumps was automatically regulated and regularly checked.

As temperature was the stressing factor, special attention has been paid to its control. In particular, the stainless-steel frame of the selectiostats were designed to reduce thermal inertia of cultures as much as possible that allowed a rapid circulation of fresh water whose temperature was regulated with a programmable cryostat (Lauda Proline RP845). Temperature regime imposed to selectiostats (Table 1) followed a succession of cycles, each of them consisting in a diel alternation of 16 and $8 \mathrm{~h}$ at high (HT) and low (LT) temperatures, respectively. The low and high temperatures were chosen in such a way that the mean daily temperature remained constant throughout the whole experiment (around $24{ }^{\circ} \mathrm{C}$ ), except for the last cycle. The decision to shift from one cycle to the next one depended on the culture fitness (estimated from the growth rate), according to the "ratchet" protocol experienced by Bonnefond et al. (2017). 
Table 1: Temperature stress cycles implemented in the selectiostats during the 150 days of ALE experiment. *As the cultures collapsed on the second day of S7 cycle, they were restarted with less stressful conditions, identical to those of S5 cycle

\begin{tabular}{ccccc}
\hline Stress cycle & HT $\left({ }^{\circ} \mathrm{C}\right)$ & $\mathrm{LT}\left({ }^{\circ} \mathrm{C}\right)$ & Cycle duration (days) & Mean temperature $\left({ }^{\circ} \mathrm{C}\right)$ \\
\hline S1 & 26 & 21 & 15 & 24.33 \\
S2 & 27 & 19 & 7 & 24.33 \\
S3 & 28 & 17 & 7 & 24.33 \\
S4 & 29 & 16 & 7 & 24.67 \\
S5 & 30 & 14 & 15 & 24.67 \\
S6 & 31 & 12 & 15 & 24.67 \\
S7 & 32 & 10 & $2 *$ & 24.67 \\
S8 & 30 & 14 & 24 & 24.67 \\
S9 & 31 & 12 & 7 & 24.67 \\
S10 & 32 & 10 & 7 & 24.67 \\
S11 & 33 & 8 & 7 & 24.67 \\
S12 & 34 & 6 & 15 & 24.67 \\
S13 & 35 & 8 & 7 & 26.00 \\
\hline
\end{tabular}

A stress indicator (Fig. 2) cumulating the pressure that the cells have faced in the selectiostat from initial experimental time to current time t was calculated as the cumulated difference from the reference temperature:

$$
\varphi(T)=\int_{0}^{t} \mid T \text {-Tref } \mid d t
$$

$T$ being the temperature at time $t$ and Tref $=24{ }^{\circ} \mathrm{C}$, the nonstressful temperature chosen as reference.

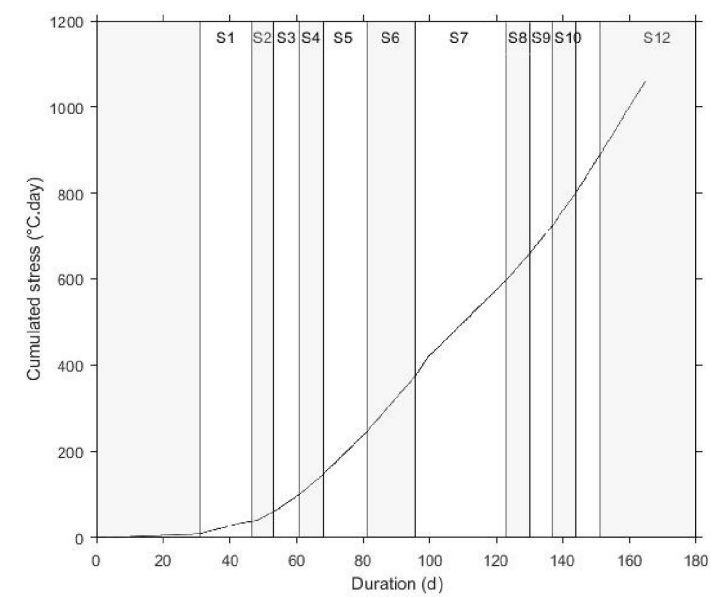

Fig. 2 Calculated stress indicator representing the cumulated stressed faced by the cells in the selectiostats during the experiment

Before use, selectiostats were sterilized with $10 \% \mathrm{HCl}$ for $20 \mathrm{~min}$ and rinsed with sterile culture medium (note that they had to be cleaned similarly 3 times during the experiment to eliminate biofouling). They were inoculated with an assemblage of the 12 strain cultures at the same concentration $(15,000$ cells $/ \mathrm{mL})$. The ODIN software (developed by Inria) allowed automatic control of the selectiostats, by recording at high frequency the parameters measured online (PAR, turbidity, pH, temperature, speed of dilution pump), and by controlling the pump speed to maintain constant turbidity (see Fig. 1).

Cell density was estimated, using a relationship established between the continuous acquisitions of turbidity and cell concentrations measured twice a month with a Multisizer 3 Beckman coulter. Cell density in continuous cultures varies accordingly to the following equation:

$$
d x / d t=\mu x-D x
$$

with $\mathrm{x}$, the cell concentration; $\mu$, the net growth rate; and $\mathrm{D}$, the dilution rate (medium flow rate divided by culture volume). When the turbidostat is at equilibrium $(d x / d t=0)$, the net growth rate equals the dilution rate

\section{Standardized measurement of the overall mixed population properties}

At the end of each stress cycle, a benchmark experiment in standardized conditions was performed to measure the properties of the overall mixed population. Triplicate batch cultures of $85 \mathrm{~mL}$ were inoculated with samples taken in each selectiostat. Duplicate batch cultures inoculated with the initial strain T. lutea CCAP 927/14 was systematically cultivated in parallel and served as reference. Indeed, CCAP $927 / 14$ is the strain of T. lutea most used in aquaculture. All the cultures were controlled with the Multi-Cultivator MC 1000-OD culturing device (Photon Systems Instruments, PSI). Growth conditions were the same as in the selectiostats, except that $\mathrm{T}^{\circ} \mathrm{C}$ was maintained constant at $20^{\circ} \mathrm{C}$, and $\mathrm{pH}$ was not regulated. Optical density (OD) was measured continuously at $720 \mathrm{~nm}$ in each flask culture to follow the growth in real time. The maximum growth rate was calculated during the exponential phase, using OD measured after $24 \mathrm{~h}$ post-inoculation and whose values did not exceed 0.3 (i.e., the value below which OD is linearly correlated with cell density).

Culture samples of $2 \mathrm{~mL}$ were filtered in duplicate on precombusted glass-fiber filters (Whatman GF/C, threshold $1.2 \mu \mathrm{m}$ ) and kept at $75{ }^{\circ} \mathrm{C}$ until particulate carbon and nitrogen analyses made with a CHN analyzer (2400 Series II CHNS/O, Perkin-Elmer).

As the biomass reached in each batch culture of the PSI Multi-Cultivator was not sufficient for accurate lipid estimation, triplicate cultures were pooled together to provide one large sample. Two sub-samples of $100 \mathrm{~mL}$ and $50 \mathrm{~mL}$, centrifuged at $3000 \mathrm{rpm}$ during $15 \mathrm{~min}\left(10{ }^{\circ} \mathrm{C}\right)$ after addition of $50 \mu \mathrm{L}$ of $\mathrm{FeCl} 3(50 \mathrm{mg} / \mathrm{mL})$ to facilitate sedimentation and formation of cell pellets, were stored at $-80{ }^{\circ} \mathrm{C}$ until extraction. 


\section{Lipid class separation}

Total lipids were extracted following the method previously described by Bligh and Dyer (1959). The total lipids were then dried using a Büchi R-200 rotavapor and then freeze-dried to remove solvent traces, then determined gravimetrically and stored at $-80{ }^{\circ} \mathrm{C}$ under nitrogen atmosphere.

The lipid classes (i.e., neutral lipids (NL), glycolipids (GL), and phospholipids (PL)) were obtained by solidphase extraction (SPE) fractionation on silica columns (Interchim, SI-S-500/6 columns) with 6 volumes of chloroform (NL), 4 volumes of acetone (GL), 6 volumes of methanol, and 2 of methanol/10\% ammonia (PL) (Vorbeck and Marinetti 1965), respectively. Each class was then dried under vacuum, determined gravimetrically, and stored at $-80{ }^{\circ} \mathrm{C}$ under nitrogen atmosphere.

\section{Fatty acids analysis}

To determine the fatty acid (FA) composition and content, the lipid class samples were methylated following the protocol described by Morrison and Smith (1964), with $7 \%$ boron trifluoride in methanol and toluene as extraction solvent for the fatty acid methyl esters (FAME). Each samplewas washed 3 times to ensure maximal FAME recovery. The FAME profiles for both phospholipids and glycolipids were obtained separately and analyzed quantitatively (fg FAME/cell) and qualitatively (\% total fatty acids, TFA). FAME were analyzed by gas chromatography (GC) on a Perkin-Elmer XL Autolab GC equipped with a flame ionization detector (FID) and a (Restek, $30 \mathrm{~m}$ length, $0.32 \mathrm{~mm}$ internal diameter). The column was operated isothermally at $182{ }^{\circ} \mathrm{C}$ while injector and detector were maintained at $250{ }^{\circ} \mathrm{C}$. Helium was used as carrier gas at $7 \mathrm{psig}$. The variability was $3 \%$ for major components, $<$ $9 \%$ for intermediate ones, and $25 \%$ for minor components $(<0.5 \%$ of total FAME); commercial nonadecanoic acid (19:0) in chloroform was co-injected with FAME sample for FA quantification.

\section{DNA extraction and sequencing}

The cell pellets of samples TL1 and TL2 stress cycles S1, $\mathrm{S} 8$, and final population $\mathrm{S} 12$ were stored at $-80{ }^{\circ} \mathrm{C}$ prior to DNA extraction, using a phenol-chloroform method described by $\mathrm{Hu}$ et al. (2004). Sequencing was carried out with an Illumina HiSeq3000, with 20 to $30 \mu \mathrm{g}$ of DNA, by the genomic platform GeT-PlaGe, INRA (Toulouse, France). The libraries were realized in TrueSeq Illumina kit $(2 \times 150$ pairedend sequenced for a DNA fragment of $430 \mathrm{pb}$ on average). Raw data was filtered to first remove all Illumina residual adapters, and to finish keep the reads with sufficient quality. It was then treated with softwares TrimGalore and home-made script to discard all reads with a mean sequencing score under Q30 or a length shorter than $120 \mathrm{pb}$. After filtering, more than $80 \%$ of the total reads were kept for each sample. The version 2.0 of reference genome of Tisochrysis lutea (approx. $85 \mathrm{Mb}$ ) (Berthelier et al. 2018) was used to map the samples' genomes with BWA-MEM software (run with default parameters). For all samples, more than $86 \%$ of the pairreads were aligned properly and in average 225 depth of sequencing genome were obtained for each sample. A search for single-nucleotide polymorphism (SNPs) was performed with Freebayes software (minimal alleles' depth 20, minimal frequency in total reads $5 \%$, pooledcontinuous option) (Garrison and Marth 2012). The results were edited using a home-made script.

To identify the strains coexisting in the cultivated population during the ALE experiment, each of the 12 initial strains were sequenced independently using the same protocol. Some specific SNPs were identified for each of them. These SNPs were used to allow the identification of the strains actually represented in the cultures during the experiment with a cross-comparison of the SNPs in each strain and the two cultivated populations. A presence index was calculated for each initial strain, by dividing its number of SNPs with the total number of SNPs.

\section{Results}

\section{Overall evolution in the selectiostat}

An example of the monitoring of selectiostat TL1 over 1 week is presented in Fig. 3 .

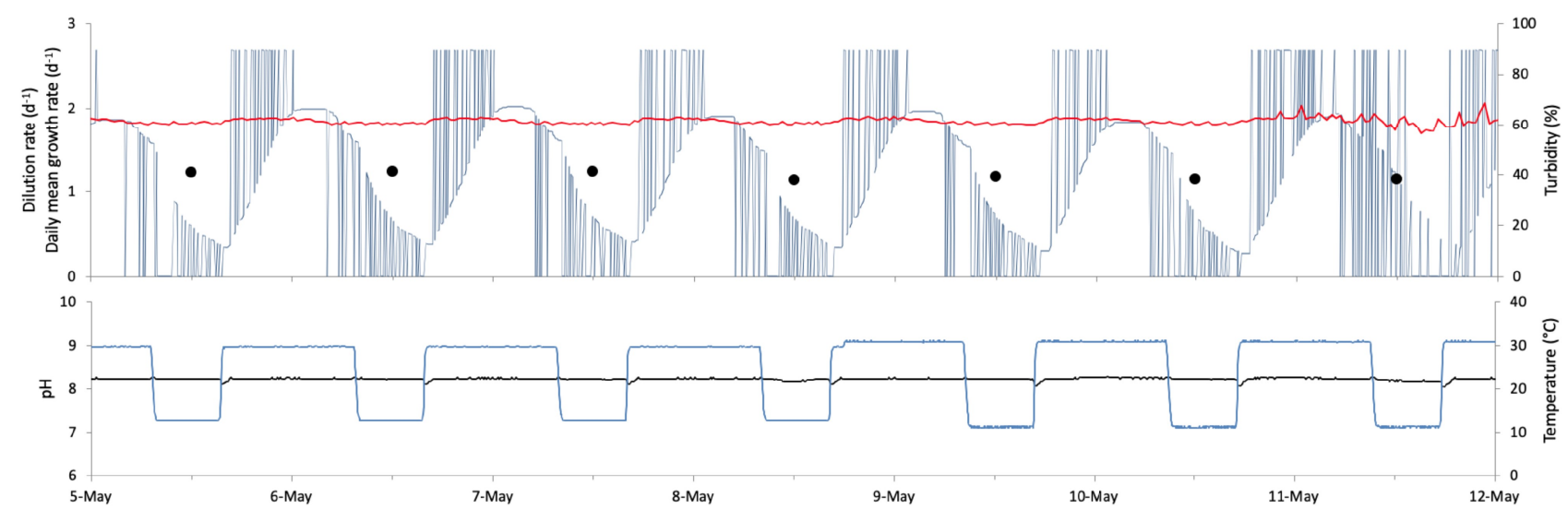

Fig. 3 Monitoring of selectiostat TL1 during 1 week. Upper graph, turbidity in red, dilution rate in blue, andmean daily growth rate in black dots. Lower graph, $\mathrm{pH}$ in black, and temperature in blue 
Turbidity was well regulated by the automatic activation of the peristatic pump, $\mathrm{pH}$ was stable, and temperature variations rapidly tracked the desired pattern between low and high temperatures, meaning that the temperature regulation through the cryostat was efficient. At the beginning of each high temperature (HT) period, the pump regularly reached its maximum speed to counterbalance growth and keep the turbidity stable by diluting the selectiostat. The pump stabilized when half of the HT period was reached, which implies that culture growth slowed down. Finally, the pump activity reduced, with frequent stops, from $\frac{3}{4}$ of the HT period and throughout the whole LT period. During that time, the growth of the culture is therefore very limited, as little dilution of the culture is then necessary to maintain a constant turbidity.

\section{Phenotype evolution in standardized culti- vation conditions}

\section{Growth rate}

The recorded growth rates estimated in the standardized benchmark conditions $(0.79 \pm 0.17$ /day $)$ are consistent with values reported by Bonnefond et al. (2017), who obtained growth rates ranging between 0.5 and 0.6 /day at $20{ }^{\circ} \mathrm{C}$, and up to 1.2 / day at the optimal growth temperature of the cultures. Growth rate (Fig. 4) was normalized according to the reference. The variations are slightly more important for culture TL1 than for TL2 (0.87-1.31 and $0.88-1.18$, respectively). However, a $\mathrm{T}$ test comparing the growth rate of each culture with the control shows no significant difference (pvalues 0.66 for TL1 and 0.76 for TL2). This shows that the selection process did not affect the overall growth rate of the population throughout the experiment and contrasts therefore with previous results obtained on mono-strain experiments (Bonnefond et al. 2017; Fu et al. 2012). A possible explanation could be that different sub-populations are coexisting and alternatively growing depending on the conditions, thus reducing the growth rate variations.

\section{Nitrogen content}

Nitrogen content in the cells tended to slightly decrease during the experiment while carbon content doubles and stabilizes from stress cycle S5 onwards for TL1 and S8 for TL2 (supplementary data, figure S2), leading to an overall increase in $\mathrm{C} / \mathrm{N}$ ratio despite the strong variability between cycles. This increase is consistent with the cell volume increase in both cultures from stress cycle S6 onwards (Table 2). The slight evolution of cell nitrogen content, as the variability of the normalized $\mathrm{C} / \mathrm{N}$ ratio (Fig. 4), could be linked to differential growth rates or result from different stresses when inoculating the benchPSI Multi-Cultivator device. After 7 days, the initial delay may have induced spreading of the time when cells are $\mathrm{N}$ limited, and thus, different $\mathrm{N}$ limitations were reached in the control or in the cultures.

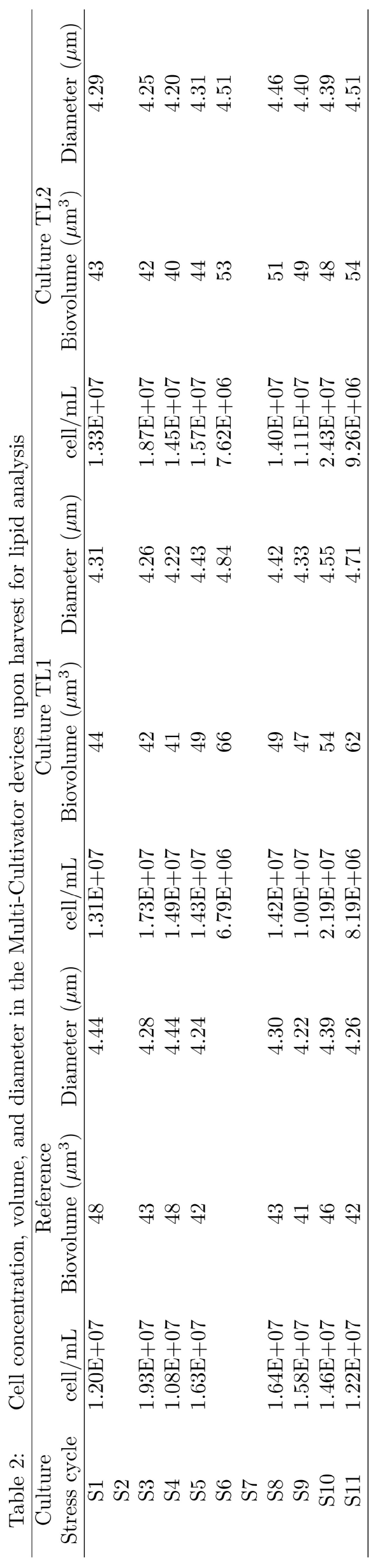



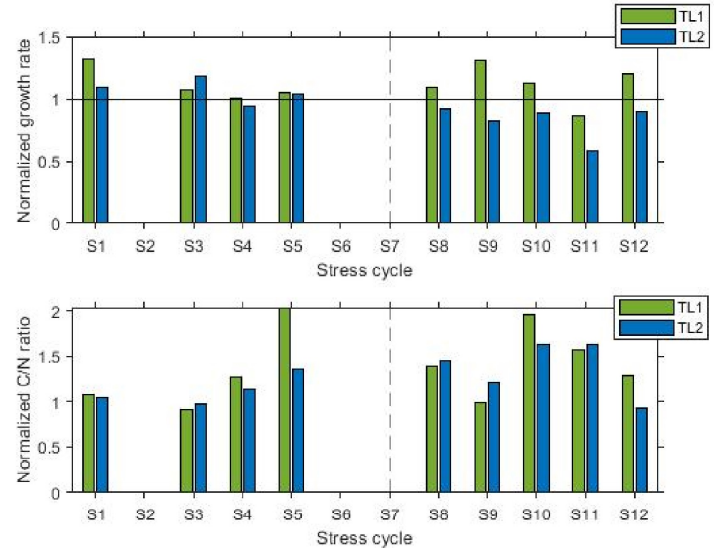

Fig. 4 Normalized growth rates* and normalized C/N ratio* of cultures in standardized conditions (PSI Multi-Cultivator device) throughout the experiment, triplicates. No data for stress cycles S2 and S6; cultures collapsed during stress S7. *Calculated as fold change in growth rate or $\mathrm{C} / \mathrm{N}$ ratio normalized to the reference

\section{Total lipid content and lipid classes}

The mass of total lipid per cell gradually increased along the selection process. From stress cycle S1 to S12, a pro- gressive enrichment of total lipid content per cell was observed. The gain between two successive stress cycles from S3 to S11 ranged from 8 to $20 \%$. This led in fine to a cellular lipid content increased by 2.1 for TL1 and 1.8 for TL2, whereas the reference culture stayed constant at an average value of $6.16 \pm 0.62 \mathrm{pg} /$ cell (Fig. 5). The cell lipid contents of both cultures are not significantly different ( $p$ value of 0.32 ). The lipid productivity showed the same increasing trend. At the end of the experiment, total and neutral lipid contents were similar to those measured by Da costa et al. $(2016,2017)$ on their lipid-rich T. lutea strain (11.2 pg/cell and $4.3-5.1 \mathrm{pg} /$ cell, respectively) in similar growing conditions (continuous $200 \mu \mathrm{mol}$ photons $/ \mathrm{m}^{2} / \mathrm{s}$ white light, $20-23{ }^{\circ} \mathrm{C}$, Conway medium).

Cellular lipid content for each lipid class (5.7-7.8 $\mathrm{pg} /$ cell for total lipids, 3.1-7.5 pg/cell for neutral lipids, 1-3.1 pg/cell for phospholipids, and 1-2.4 pg/cell for glycolipids) was slightly higher than those measured (via HPTLC) by Carrier et al. (2018) (3-6 pg/cell for total lipids, $0.4-3.1 \mathrm{pg} /$ cell for triacylglycerol, $0.9-1.3 \mathrm{pg} /$ cell for phospholipids, and $1.4-1.7 \mathrm{pg} /$ cell for glycolipids). The quantitative measurements of each major lipid class after fractionation (Fig. 5) show a cellular enrichment of every class from stress cycle S5 onwards.
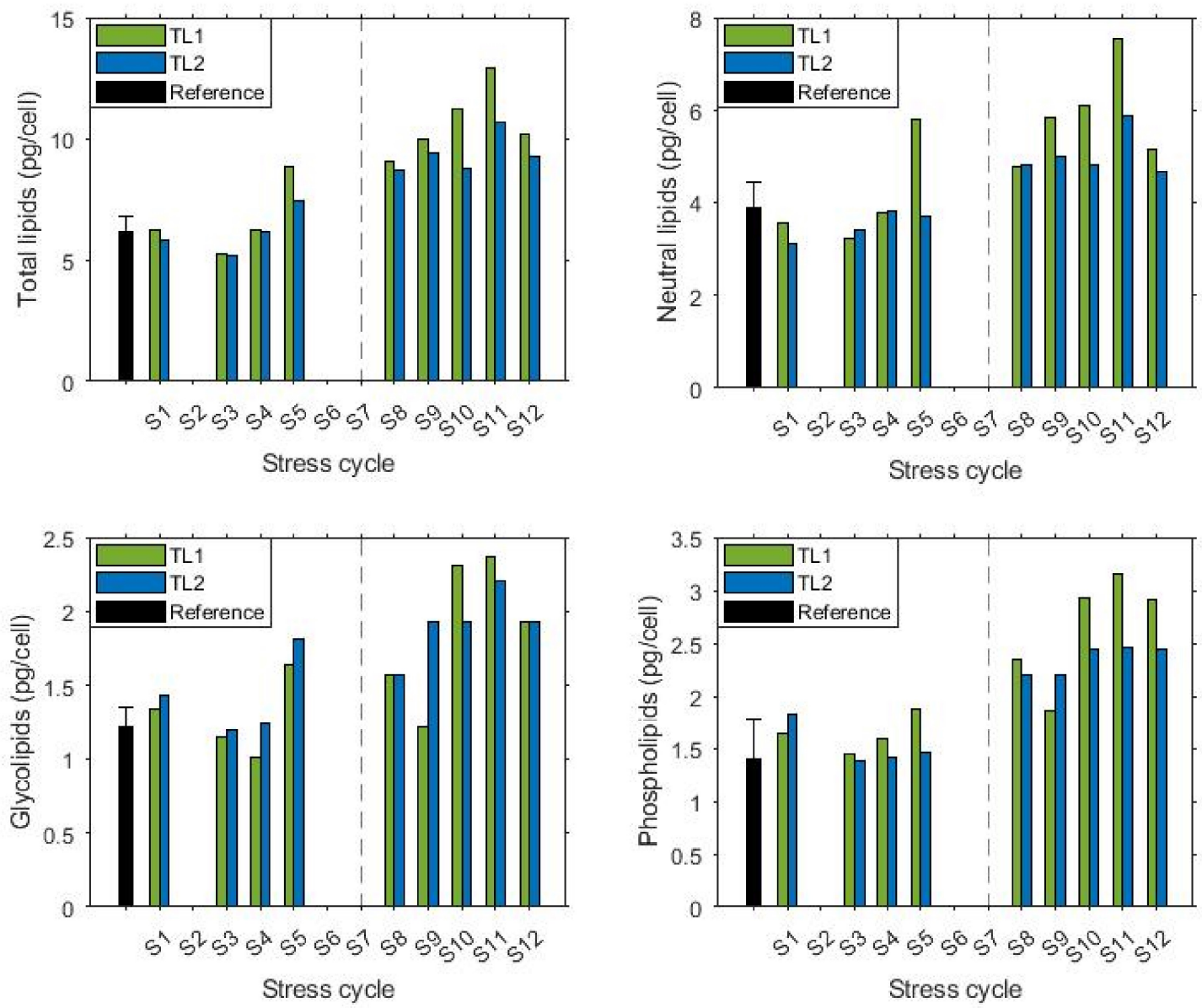

Fig. 5 Evolution of cellular total lipid and lipid class contents (pg/cell) throughout the stress cycles; cultures in standardized cultivation conditions in the Multi-Cultivator. No data for stress cycles S2 and S6; cultures collapsed during stress S7. Reference is an average of 9 measurements on independent cultures 


\section{Fatty acids composition}

The main fatty acids partitioned to phospholipids were 14:0, 16:0, 18:1 n-9, 18:3 n-3, 18:4 n-3, and 22:6 n-3 in the 2 stressed and reference cultures. The same fatty acids were incorporated into glycolipids together with 18:2 n-6. Overall, fatty acid profiles of polar lipids (GL + PL) (Table 3) were similar to those reported by Marchetti et al. (2018), and closely similar to total fatty acid profile $(16 \%$ for $14: 0,14.5 \%$ for $16: 0,4.2 \%$ for $16: 1 \mathrm{n}-7,20.1 \%$ for $18: 1$ $\mathrm{n}-9,3.6 \%$ for $18: 3 \mathrm{n}-3,17.4 \%$ for $18: 4 \mathrm{n}-3,2.5 \%$ for $18: 5$ $\mathrm{n}-3$, and $8.5 \%$ for $22: 6 \mathrm{n}-3$ ) obtained by Volkman et al. (1989). However, they differed from the profiles obtained by Renaud et al. (1995) who cultivated T. lutea at 15, 20, 25 , and $30{ }^{\circ} \mathrm{C}$. They obtained significantly higher amounts of 14:0 (except at $15{ }^{\circ} \mathrm{C}$ ), 18:4 n-3 $(>20 \%,>22 \%$, respectively) and 18:5 n-3 but only at the lowest temperature $\left(15{ }^{\circ} \mathrm{C}\right)$. On the other hand, they observed lower amounts of 16:0, 18:1 n-9, 18:2 n-6, 22:5 n-6, and DHA except at $15{ }^{\circ} \mathrm{C}(<9.6 \%,<8.7 \%,<4.8 \%,<1 \%$, and $<9.7 \%$, respectively). However, Renaud et al. (1995) and Volkman et al. (1989) studied total lipids, not polar lipids; the observed variations can therefore be caused by the specific fatty acid composition of polar lipids, which can greatly differ from the overall fatty acid composition.

\begin{tabular}{|c|c|c|c|c|c|}
\hline Fatty acid (\% TFA) & Reference & TL1-S1 & TL1-S12 & TL2-S1 & TL2-S12 \\
\hline $14: 0$ & $13.99 \pm 3.23$ & 15.21 & 11.75 & 16.94 & 8.01 \\
\hline ISO15:0 & $0.22 \pm 0.10$ & 0.22 & 0.09 & 0.12 & 0.04 \\
\hline $15: 0$ & $0.55 \pm 0.43$ & 0.70 & 0.16 & 0.29 & 0.13 \\
\hline ISO17:0 & $0.19 \pm 0.16$ & 0.30 & 0.22 & 0.00 & 0.39 \\
\hline $16: 0$ & $15.26 \pm 2.34$ & 13.58 & 14.91 & 16.39 & 9.61 \\
\hline $17: 0$ & $0.25 \pm 0.21$ & 0.35 & 0.06 & 0.25 & 0.06 \\
\hline 18:0 & $0.54 \pm 0.18$ & 0.52 & 0.31 & 0.35 & 0.30 \\
\hline Total SFAs & $31.25 \pm 5.14$ & 31.02 & 27.69 & 37.75 & 18.67 \\
\hline $14: 1 \mathrm{n}-7$ & $0.13 \pm 0.13$ & 0.36 & 0.16 & 0.16 & 0.00 \\
\hline $14: 1 \mathrm{n}-5$ & $0.23 \pm 0.09$ & 0.35 & 0.21 & 0.50 & 0.22 \\
\hline $16: 1 n-7$ & $3.71 \pm 0.25$ & 3.27 & 3.72 & 3.62 & 4.01 \\
\hline $16: 1 \mathrm{n}-5$ & $0.15 \pm 0.10$ & 0.06 & 0.05 & 0.21 & 0.00 \\
\hline $17: 1 \mathrm{n}-10$ & $0.08 \pm 0.07$ & 0.13 & 0.03 & 0.14 & 0.08 \\
\hline $18: 1 n-9$ & $16.22 \pm 3.71$ & 10.63 & 20.75 & 12.81 & 22.35 \\
\hline $18: 1 \mathrm{n}-7$ & $0.35 \pm 0.22$ & 0.40 & 1.17 & 0.35 & 0.00 \\
\hline $18: 1 \mathrm{n}-5$ & $0.12 \pm 0.11$ & 0.00 & 0.05 & 0.00 & 0.00 \\
\hline $22: 1$ n-13 + 11 & $0.09 \pm 0.13$ & 0.13 & 0.00 & 0.15 & 0.17 \\
\hline Total MUFAs & $21.32 \pm 3.62$ & 15.42 & 26.18 & 18.07 & 26.87 \\
\hline $16: 2 n-6$ & $0.20 \pm 0.17$ & 0.22 & 0.03 & 0.25 & 0.00 \\
\hline $16: 2 n-4$ & $1.05 \pm 0.12$ & 0.77 & 0.76 & 1.18 & 0.82 \\
\hline $18: 2 n-6$ & $4.48 \pm 0.94$ & 4.37 & 4.94 & 3.90 & 6.10 \\
\hline $20: 2 \mathrm{n}-6$ & $0.14 \pm 0.04$ & 0.13 & 0.08 & 0.15 & 0.09 \\
\hline $16: 3 n-4$ & $0.07 \pm 0.02$ & 0.11 & 0.05 & 0.13 & 0.07 \\
\hline $16: 3 n-3$ & $0.26 \pm 0.26$ & 0.29 & 0.09 & 0.16 & 0.13 \\
\hline $18: 3$ n-6 & $0.16 \pm 0.08$ & 0.06 & 0.14 & 0.08 & 0.22 \\
\hline $18: 3 n-3$ & $6.47 \pm 0.56$ & 6.97 & 7.90 & 6.75 & 7.94 \\
\hline $20: 3 \mathrm{n}-3$ & $0.16 \pm 0.06$ & 0.04 & 0.00 & 0.05 & 0.11 \\
\hline $16: 4 n-3$ & $0.06 \pm 0.03$ & 0.03 & 0.05 & 0.09 & 0.02 \\
\hline $16: 4 n-1$ & $0.16 \pm 0.08$ & 0.18 & 0.10 & 0.08 & 0.11 \\
\hline $18: 4 n-3$ & $14.68 \pm 2.99$ & 16.40 & 15.79 & 14.82 & 23.64 \\
\hline $20: 4$ n-6 & $0.10 \pm 0.07$ & 0.12 & 0.05 & 0.06 & 0.04 \\
\hline $22: 4$ n-6 & $0.09 \pm 0.06$ & 0.05 & 0.00 & 0.18 & 0.05 \\
\hline $18: 5 n-3$ & $3.77 \pm 0.69$ & 5.57 & 3.25 & 3.35 & 5.50 \\
\hline $20: 5 n-3$ & $0.26 \pm 0.10$ & 0.45 & 0.24 & 0.35 & 0.18 \\
\hline $21: 5 \mathrm{n}-3$ & $0.64 \pm 0.26$ & 0.78 & 0.55 & 0.50 & 0.58 \\
\hline $22: 5 n-6$ & $2.30 \pm 0.44$ & 2.39 & 1.87 & 2.27 & 1.04 \\
\hline $22: 5 \mathrm{n}-3$ & $0.13 \pm 0.07$ & 0.14 & 0.06 & 0.23 & 0.07 \\
\hline 22:6 n-3 & $11.86 \pm 1.92$ & 14.08 & 10.12 & 11.94 & 7.12 \\
\hline Total PUFAs & $47.43 \pm 6.63$ & 53.55 & 46.13 & 44.18 & 54.47 \\
\hline Others & 0.89 & 0.61 & 0.28 & 1.21 & 0.81 \\
\hline Total n-3 PUFAs & $39.29 \pm 6.95$ & 44.77 & 38.05 & 39.23 & 45.29 \\
\hline Total n-6 PUFAs & $7.46 \pm 1.80$ & 7.356309 & 7.11 & 4.89 & 7.54 \\
\hline n-3/n-6 ratio & 5.13 & 6.09 & 5.35 & 5.55 & 6.00 \\
\hline
\end{tabular}

Table 3 Proportions of fatty acids in the polar lipids representing more than $0.1 \%$ TFA at the beginning and the end of the experiment. Cultures in standardized conditions (PSI Multi-Cultivator device). Reference is an average of 8 measurements on independent cultures. Major FAME ( $>2 \%$ TFA) of the fatty acids profile are emphasised in italics 
The fatty acid profiles at S1 were similar to the reference for both cultures, except 18:1 n-9 which is slightly lower compared to the reference culture $(10.63 \%$ and $12.81 \%$ for TL1 and TL2, respectively, $16.22 \pm 3.71 \%$ for the reference). Similarly, the total saturated fatty acid (SFAs) and PUFA proportions were similar; the lower total monounsaturated fatty acid (MUFAs) in the cultures is linked to their lower 18:1 n-9 content. At S12, the selection process seemed to have impacted the fatty acid composition, especially of polar lipids. The two major SFAs (14:0 and/or 16:0) diminished while the proportion of C18-MUFAs (18:1 n-9) and most C18-PUFAs (18:2 n-6, 18:3 n-3 and 18:4 n-3) increased. Changes in PUFAs (in particular DHA) fluctuate between the two photobioreactors.

The cellular content of DHA partitioned to polar lipids nearly tripled between the beginning and the end of the ALE experiment. It was mainly due to the important increase of the total lipid cell content. DHA increase is significant from stress cycle S8 onwards (Fig. 6). Indeed, in the first half of the experiment, before cycle S7, the DHA content partitioned to polar lipids varied between 50 and $100 \mathrm{fg} /$ cell. In the second half of the experiment, it reached $200 \mathrm{fg} /$ cell during stress cycles S8 and S9, then increased of $13 \pm 3 \%$ between stress cycles S9 to S12 for TL1, and 20 to $23 \%$ for TL2. This difference could result from the intense stress during cycle $\mathrm{S} 7$ which led to the culture crash. It may have caused a stronger selection, and possibly the emergence of a sub-population with a higher DHA content.

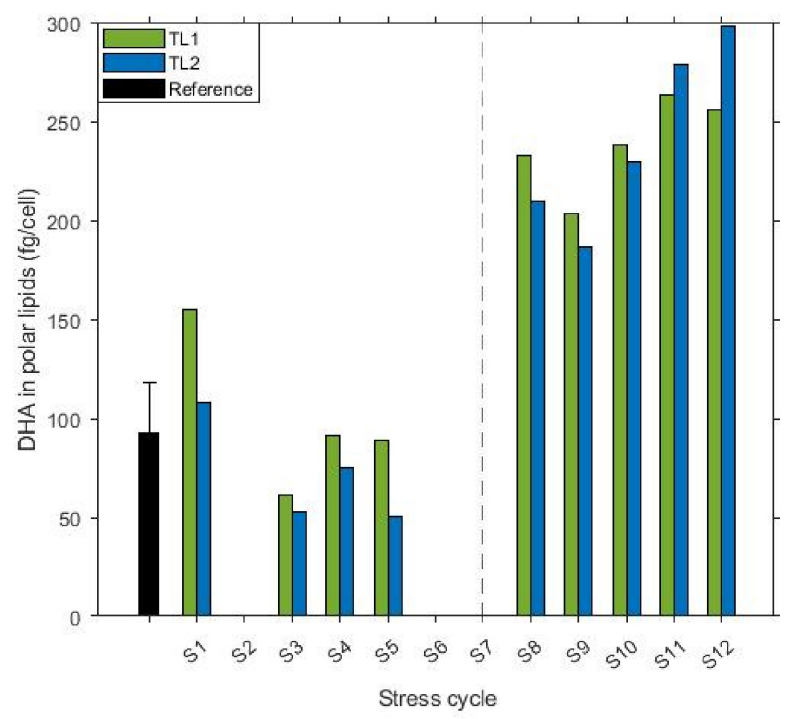

Fig. 6 Evolution of cellular DHA content partitioned to polar lipids. No data for stress cycles S2 and S6; cultures collapsed during stress S7. Reference is an average of 9 measurements on independent cultures

\section{Genomic analysis}

Samples for genomic analysis were taken and analyzed at the end of stress cycle S1, S8, and S12. Through the SNP search, mutations corresponding to a total change of genotype (disappearance of the initial genotype) were detected in both cultures and for each sequenced sample. It demonstrates an evolution of the initial population rather than a simple selection of some initial characteristics.

The overall presence indexes were calculated from crosscomparison of the global SNPs with the initial ones. Presence indexes inferior to 0.15 mean that the corresponding strain is likely to be absent from the population. They ranged between 0.08 and 0.16 except for strains CCMP 463 (0.40) and RCC 1344 (0.50) (Table 4). This demonstrates that these two strains dominate the population, since the beginning of the experiment. Both are originated from low latitudinal coastal areas from north hemisphere: CCMP 463 was isolated in the Caribbean archipelago (Turks and Caicos Islands) and RCC 1344 along the Spanish Atlantic coast (Bendif et al. 2013). This data suggests that, out of the initial twelve-strain assemblage, only CCMP 463 and RCC 1344 compose the population undergoing the stressed cultivation and the final population of T. lutea. The 10 other strains were cleaned up during the build-up phase (batch growth and turbidostat stabilization) and during first stress cycle (S1) of the experiment.

Total mutation and selection events detected at stress cycle S1, S8, and S12 stayed quite stable for both cultures throughout the experiment $(5874,4743$, and 4783 for TL1 and 4870,3725 , and 5232 for TL2, respectively). This is surprising considering the culture crash at stress cycle S7, with the loss of a large fraction of the cells and the observed change in the lipid phenotype, suggesting that this event had little impact on the genomic diversity of the population.

Few SNPs led to a complete change of the genotype, with the disappearance of the initial one: 49 for culture TL1 and 17 for culture TL2. Further analyses on those specific mutations were carried out to determine whether they could be partially responsible for the phenotype changes observed on the final populations. For culture TL2, 2 out of the 17 SNP mutations were localized in coding regions, and 9 out of 49 for culture TL1. Putativeassociated functionality search on these genes remains on the prospective side, as very little is known on the function of genes in T. lutea. Researches of homologous genes in Uniprot database did not lead to associated functionality for 7 of the genes; 2 could be GAG-POL transposons. The last 4, all detected in culture TL1, could be impacting a ClpC protease, a dihydrolipoamide acyltransferase part of the pyruvate deshydrogenase complex, a trehalosephosphate synthase, and a ribonucleotide-diphosphate reductase. Extensive studies of these candidate genes must be conducted to confirm their potential roles. Especially, the $\mathrm{ClpC}$ protease could act as a chaperone during heat shocks by targeting denatured proteins and addressing them for degradation (Uniprot database, identified function in Staphylococcus aureus). If that role was confirmed in $T$. lutea, it would be very interesting to investigate how it impacts the operation of the protein and potentially link it to the dynamic stress protocol. 


\section{Discussion}

In the selectiostat devices, the cell growth rate presented daily oscillations (Fig. 3), with constant mean daily growth rate. This behavior was different from observations on singlestrain continuous cultures by Bonnefond et al. (2017). It might be due to the coexistence of several sub-populations in the selectiostats, which grow alternatively and thus reduce the apparent variations in the growth rate. A closer look at daily growth rate pattern shows a clear phasing with temperature oscillations. This is probably due to the synchronization of cell division with temperature as already observed in D. salina (Bonnefond et al. 2016). Most of the cells divide at the same time during the first half of each HT period, generating an increase in turbidity.

The selection process occurring in the selectiostats seemed to favorably impact lipid metabolism with higher lipid content in all lipid classes; however, polar lipids doubled while neutral lipids increased only by 1.5 -fold. This is also visible in the calculated proportions (supplementary data, figure S1), which show a slight decrease for neutral lipids and concomitantly a slight increase in phospholipids while glycolipids remain stable throughout the experiment. These data, and notably the polar lipids changes, are consistent with themeasured increase of the cell volume during the experiment (Table 2). Indeed, this means that more cell membranes are produced, thus increasing the quantity of phospholipids, but not necessarily more chloroplasts since glycolipids compose thylakoidal membranes and not cell membranes. The accumulation of a pool of MUFAs and PUFAs partitioned to polar lipids inside the cell is also likely to allow quicker membrane acclimation to temperature fluctuations. Lipid accumulation (neutral and polar) might also contribute to the cell metabolic response for keeping cellular redox homeostasis. Indeed, fatty acids are highly reduced molecules, which can be used as an alternative sink for electrons produced by the photosynthetic chains, especially at colder temperature, avoiding an over-reduction of the NADP pool (Zhang et al. 2016).

When growth temperature of $T$. lutea was increased from 10 to $20{ }^{\circ} \mathrm{C}$, Thompson et al. (1992) measured a strong metabolic shift from 16:1 n-7 to $18: 1$ n-9 and greater production of FA 18:5 n-3. This acclimation strategy is coherent with the lipid adaptation obtained in the ALE experiment. Since 18:1 n-9 is the first unsaturated fatty acid in the PUFA biosynthesis pathway, this could be a metabolic shift of the cells to prioritize the PUFA biosynthesis pathway over the one of SFAs. The rationale behind the 18:5 n-3 shifts is unclear as it diminishes of $42 \%$ in TL1 and increases of $64 \%$ in TL2 between stress cycle S1 and S12. However, most C18-PUFAs (18:2 n- 6, 18:3 n-3, and 18:4 n-3) increased from cycles S1 to S12. These modifications in the FA profile, and more specifically the accumulation of $\mathrm{C} 18$ fatty acids, along with the increase in cell bio- volume, suggest a strategy to maintain membrane fluidity for adapting to fluctuating temperatures. It is consistent with previous observations and conclusions when studying both cell adaptation with a similar approach (Bonnefond et al. 2017) and cell acclimation (Renaud et al. 1995).

Our new population of T. lutea fits the major criteria to be used as feed for bivalve larvae (Rico-Villa et al. 2006 ), i.e., a rapid growth and an easy cultivation (these parameters were not impacted by the evolution process of the experiment). The larger cell diameter (around $5 \mu \mathrm{m}$ with an increase detected from stress cycle S6 onwards, Table 2) is likely to present an improved nutrition value compared to T. lutea wild-type. Indeed, the observations made by Da Costa et al. (2016) on the growth and survival of $C$. gigas larvae fed with $T$. lutea suggest that a high level of NL within total lipids impedes the larvae's survival. The new mixed-population, with higher polar lipids' content compared to NL, is likely to provide a more balanced lipid source as the fatty acid profile of polar lipids (see below) has hardly been affected. Moreover, the importance of PUFAs for the development of $C$. gigas larvae has been extensively studied (Chu and Webb 1984; Rico-Villa et al. 2006; Da Costa et al. 2016). It was shown that EPA and DHA contents in the range of $7-17 \%$ and $7-14 \%$ of TFA, respectively (Rico-Villa et al. 2006), were necessary to ensure a good larval development. Tisochrysis lutea produces only traces of EPA but itsDHA content iswithin the necessary proportion range. However, the cells of our new population are richer in total lipids compared to the reference; therefore, it could be a better source of DHA for oysters.

The presence indexes calculated from the SNPs are representative of the probability to exist in the evolving population. It therefore does not mean that the two remaining strains were equally represented in the population. Moreover, as the first sampling was done at the end of S1, it is not possible to know which of the 10 strains disappeared during the build-up phase (because of a slower growth), and which disappeared during the first stress cycle (because they could not acclimate). Strain CCMP463 presented the same pattern in both culture during the experiment: the presence index increased between $\mathrm{S} 1$ and $\mathrm{S} 8$, then stabilized between S8 and S12. On the other hand, strain RCC 1344 behaved slightly differently in both cultures. Its presence index increased from 0.11 to 0.43 in culture TL1, but varied between 0.42 and 0.45 in culture TL2. This deviation could be a possible explanation for the differences measured on the fatty acid profiles in both cultures.

Finally, Costas et al. (2014) suggested that I. galbana wild strain had a "greater genetic variability in fitness," with a high adaptive potential to thermal stress compared to the other species they studied. Our data tends to confirm this result for T. lutea, as the final population was able to grow outside its initial thermal niche. 
Table 4: Presence index of the different strain in the cultivated population, calculated from the SNPs, at stress cycles S1, S8, and S12. Major presence indexes $(>0.10)$ are emphasised in italics

\begin{tabular}{ccccccc}
\hline Strain & TL1-S1 & TL1-S8 & TL1-S12 & TL2-S1 & TL2-S8 & TL2-S12 \\
\hline CCAP 927/14 & 0.008 & 0.037 & 0.028 & 0.031 & 0.054 & 0.028 \\
CCMP 463 & 0.174 & 0.315 & 0.353 & 0.190 & 0.370 & 0.375 \\
RCC 179 & 0.012 & 0.036 & 0.036 & 0.036 & 0.060 & 0.060 \\
RCC 1344 & 0.112 & 0.427 & 0.420 & 0.448 & 0.420 & 0.413 \\
RCC 3691 & 0.013 & 0.033 & 0.013 & 0.033 & 0.033 & 0.007 \\
RCC 3692 & 0.023 & 0.068 & 0.008 & 0.030 & 0.015 & 0.008 \\
RCC 3693 & 0.056 & 0.028 & 0.065 & 0.046 & 0.019 & 0.028 \\
RCC 3699 & 0.039 & 0.072 & 0.02 & 0.033 & 0.039 & 0.013 \\
Argenton 1998 & 0.030 & 0.060 & 0.045 & 0.015 & 0.015 & 0.045 \\
IFMG & 0.028 & 0.037 & 0.009 & 0.019 & 0.019 & 0.009 \\
NIVA 4-91 & 0.026 & 0.017 & 0.009 & 0.017 & 0.017 & 0.026 \\
S5-1\% & 0.036 & 0.018 & 0.018 & 0.00 & 0.018 & 0.009 \\
\hline
\end{tabular}

This study demonstrated the efficiency of ALE with an enhanced population of T. lutea enriched in PUFA-rich polar lipids. Two strains out of the 12 composing the initial population turn out to survive the adaptation experiment. The observed mutations suggest that evolution took place upon selection. The lipid phenotype drastically changed during the second half of the experiment, with a twofold increase in total lipid content and a threefold increase in the DHA partitioned to polar lipids. As growth rate was not affected, the gain in lipid and DHA is directly a gain in productivity.

There is currently a lack of data to assess the stability of the metabolic changes after months of cultivation conditions back to normal. A possibility to keep them for longer periods of time, while continuing to improve their performance, is to maintain the selection pressure, but this strategy is expensive, with high manpower costs. Screening the population in order to isolate the clones presenting the desired phenotype will prevent the strain to be outcompeted by individuals in minority. On top of this, cryopreserving the strain will definitely guaranty constant properties when inoculating a new reactor.

Supplementary Information The online version contains supplementary material available at https://doi.org/10.1007/s00253-020-11000-4.

Acknowledgments This work was performed, in partnership with the SAS PIVERT, within the frame of the French Institute for the Energy Transition (Institut pour la Transition Energétique (ITE) P.I.V.E.R.T. (www.institut-pivert.com) selected as an Investment for the Future ("Investissements d'Avenir"). This work was supported, as part of the Investments for the Future, by the French Government under the reference ANR-001-01.

Authors' contributions MG, AT, and EP designed and conducted the experiments. MG,MB, and GC analyzed data. MG wrote the manuscript. GC, FG, OB, and AS corrected the manuscript. All authors read and approved the manuscript.

Funding This study was funded by P.I.V.E.R.T. (GENESYS program - WP4P5-73-01).

Data availability Not applicable

\section{Compliance with ethical standards}

Conflict of interest The authors declare that they have no conflicts of interest.

Code availability Not applicable

Ethical approval This article does not contain any studies with human participants or animals, performed by any of the authors.

\section{References}

Ackman R, Tocher C, McLachan J (1968) Marine phytoplankter fatty acids. J Fish Res Board Can 25:1603-1620. https://doi.org/10.1139/ f68-145

Bellou S, Baeshen MN, Elazzazy AM, Aggeli D, Sayegh F, Aggeli G (2014) Microalgal lipids biochemistry and biotechnological perspectives. Biotechnol Adv 32:1476-1493. https://doi.org/10.1016/j.biotechadv.2014.10.003

Bendif E, Probert I, Schroeder DC, de Vargaas C (2013) On the description of Tisochrysis lutea gen. nov. sp. No. and Isochrysis nuda sp. Nov. in Isochrysidales, and the transfer of Dicrateria to the Prymnesiales (Haptophyta). J Appl Phycol 25:1763-1776. https:// doi.org/10.1007/s10811-014-0284-8

Berthelier J, Casse N, Daccord N, Jamilloux V, Saint-Jean B, Carrier G (2018) A transposable element annotation pipeline and expression analysis reveal potentially active elements in the microalga Tisochrysis lutea. BMC Genomics 19:378. https://doi.org/10.1186/ s12864-018-4763-1

Bligh EG, Dyer WJ (1959) A rapid method of total lipid extraction and purification. Can J Biochem Physiol 37:911-917. https://doi.org/10.1139/y59-099

Bonnefond H, Moelants N, Talec A, Bernard O, Sciandra A (2016) Concomitant effects of light and temperature diel variations on the growth rate and lipid production of Dunaliella salina. Algal Res 14: 72-78. https://doi.org/10.1016/j.algal.2015.12.018

Bonncfond H, Grimaud G, Rumin J, Bougaran G, Talcc A, Gachelin M, Boutoute M, Pruvost E, Bernard O, Sciandra A (2017) Continuous selection pressure to improve temperature acclimation of Tisochrysis lutea. PLoS One 12(9):e0183547. https://doi.org/10. 1371/journal.pone.0183547

Carrier G, Baroukh C, Rouxel C, Duboscq-Bidot L, Schreiber N, Bougaran G (2018) Draft genomes and phenotypic characterization of Tisochrysis lutea strains. Towards the production of domestical strains with high added value. Algal Res 29:1-11. https://doi.org/10. 1016/j.algal.2017.10.017

Chu F-LE, Webb KL (1984) Polyunsaturated fatty acids and neutral lipids in developing larvae of the oyster Crassostrea virginica. Lipids 19:815-820. https://doi.org/10.1007/BF02534509

Costas E, Baselga-Cervera B, Garcia-Balboa C, Lopes-Rodas $\mathrm{V}$ (2014) Estimating the genetic capability of different phytoplankton organisms to adapt to climate warming. Oceanogr 2. https://doi.org/10. 4172/2332-2632.1000123 
Da Costa F, Petton B, Mingant C, Bougaran G, Rouxel C, Quéré C, Wikfors GH, Soudant P, Robert R (2016) Influence of one selected Tisochrysis lutea strain rich in lipids on Crassostrea gigas larval development and biochemical composition. Aquac Nutr 22:813- 836. https://doi.org/10.1111/anu.12301

Da Costa F, Le Grand F, Quéré C, Bougaran G, Cadoret J-P, Robert R, Soudant P (2017) Effects of growth phase and nitrogen limitation on biochemical composition of two strains of Tisochrysis lutea. Algal Res 27:177-189. https://doi.org/10.1016/j.algal.2017.09.003

Dragosits M, Mattanovich D (2013) Adaptive laboratory evolution - principles and applications for biotechnology. Microbial cell factories 12:64. https://doi.org/10.1186/1475-2859-12-64

FAO (2017) The future of food and agriculture - trends and challenges. Rome

Fu W, Gudmundsson O, Feist AM, Herjolfsson G, Brynjolfsson S, Palsson BO (2012) Maximizing biomass productivity and cell density of Chlorella vulgaris by using light-emitting diode-based photobioreactor. Journal of Biotechnology 161:242-249. https:// doi.org/10.1016/j.jbiotec.2012.07.004

Fu W,GudmundssonO, PagliaG,Herjolfsson G, Andresson OS, Palsson B, Brynjolfsson S (2013) Enhancement of carotenoid biosynthesis in the green microalga Dunaliella salina with light-emitting diodes and adaptive laboratory evolution. Appl Microbiol Biotechnol 97:2395-2403. https://doi.org/10.1007/s00253-012-4502-5

Garrison E, Marth G (2012) Haplotype-based variant detection from short-read sequencing. https://arxiv.org/abs/1207.3907. Accessed July 2019

Hu Z, Zeng X, Wang A, Shi C, Duan D (2004) An efficient method for DNA isolation from red algae. J Appl Phycol 16:161-166. https://doi.org/10.1023/B:JAPH.0000048456.26639.1a

Li D, Wang L, Zhao Q, Wei W, Sin Y (2015) Improving high carbon dioxide tolerance and carbon dioxide fixation capability of Chlorella sp. by adaptive evolution. Bioresour Technol 185:269275. https://doi.org/10.1016/j.biortech.2015.03.011

Liu J, Sommerfeld M, Hu Q (2013) Screening and characterization of Isochrysis strains and optimization of culture conditions for docosahexaenoic acid production. Appl Microbiol Biotechnol 97:4785-4798. https://doi.org/10.1007/s00253-013-4749-5

Marchetti J, Da Costa F, Bougaran G, Quéré C, Soudant P, Robert R (2018) The combined effects of blue light and dilution rate on lipid class and fatty acid composition of Tisochrysis lutea. J Appl Phycol 30:1483-1494. https://doi.org/10.1007/s10811-017-1340-y

Morrison WR, Smith LM (1964) Preparation of fatty methyl esters and dimethylacetals from lipids with boron fluoride-methanol. J Lipid Res 5:600-608

Mortensen S, Borsheim K, Rainuzzo J, Knutsen G (1998) Fatty acid and elemental composition of the marine diatom Chaetoceros gracilis Schütt. Effects of silicate deprivation, temperature and light intensity. J ExpMar Biol Ecol 122:173-185. https://doi.org/10.1016/0022-0981(88)90183-9

Naylor RL, Hardy RW, Bureaus DP, Chiu A, Elliott M, Farrel AP, Forster I, Gatlin DM, Goldburg RJ, Hua K, Nichols PD (2009) Feeding aquaculture in an era of finite resources. Proc Natl Acad Sci U.S.A. 106:15103-15110. https://doi.org/10.1073/pnas.0905235106

Perrineau M-M, Zelzion E, Gross J, Price DC, Boyd J, Bhattacharya D (2013) Evolution of salt tolerance in a laboratory reared population of Chlamydomonas reinhardtii. Environ Microbiol 16 https://doi.org/10.1111/1462-2920.12372

Renaud SM, Zhou H, Parry D, Thinh L-V, Woo K (1995) Effect of temperature on the growth, total lipid content and fatty acid composition of recently isolated tropical microalgae Isochrysis sp., Nitzschia closterium, Nitzschia paleacea, and commercial species Isochrysis sp. (clone T.ISO). J Appl Phycol 7:595-602. https://doi.org/10.1007/BF00003948

Renaud SM, Thinh L-V, Lambrinidis G, Parry DL (2002) Effect of temperature on growth, chemical composition and fatty acid composition of tropical Australian microalgae grown in batch cultures. Aquaculture 211:195-214. https://doi.org/10.1016/S00448486(01)00875-4

Rico-Villa B, Le Coz J, Mingant C, Robert R (2006) Influence of phytoplankton diet mixtures on microalgae consumption, larval development and settlement of the Pacific oyster Crassostrea gigas (Thunberg). Aquaculture 256:377-388 https://doi.org/10.1016/j.aquaculture.2006.02.015

Roleda MY, Slocombe SP, Leakey RJ, Day JG, Bell EM, Stanley MS (2013) Effects of temperature and nutrient regimes on biomass and lipid production by six oleaginous microalgae in batch culture employing a two-phase cultivation strategy. Bioresour Technol 129:439-449. https://doi.org/10.1016/j.biortech.2012.11.043

Santos-Sanchez N, Valadez-Blanco R, Hernandez-Carlos B, Torres- Ariño A, Guadarrama-Mendoza P, Slals-Coronado $\mathrm{R}$ (2016) Lipids rich in $\omega 3$ polyunsaturated fatty acids from microalgae. Appl Microbiol Biotechnol 100:8667-8684 https://doi.org/10.1007/s00253-016-7818-8

Spolaore P, Joannis-Cassan C, Duran E, Isambert A (2006) Commercial applications of microalgae. J Biosci Bioeng 101:87-96. https://doi.org/10.1263/jbb.101.87

Thompson PA, Guo M-X, Harrison PJ, Whyte JN (1992) Effects of variation in temperature. II. on the fatty acid composition of eight species of marine phytoplankton. J Phycol 28:488-497. https://doi.org/10.1111/j.0022-3646.1992.00488.x

Volkman J, Jeffrey S, Nichols P, Rogers G, Garland C (1989) Fatty acid and lipid composition of 10 species of microalgae used in mariculture. J Exp Mar Biol Ecol 128:219-240. https://doi.org/10.1016/0022-0981(89)90029-4

Vorbeck ML, Marinetti G (1965) Separation of glycosyl diglycerides from phosphatides using silicic acid column chromatography. J Lipid Res 6:1-6

Walne PR (1974) Culture of bivalve molluscs. England Fishing News Books Ltd WAL

Wang D, Ju X, Zhou D, Wei G (2014) Efficient production of pullulan using rice hull hydrolysate by adaptive laboratory evolution of Aureobasidium pullulans. Bioresour Technol 164:12-19. https://doi.org/10.1016/j.biortech.2014.04.036

Wang L, Xue C,Wang L, Zhao Q,WeiW, Sun Y (2016) Strain improvement of Chlorella sp. for phenol biodegradation by adaptive laboratory evolution. Bioresour Technol 205:264-268

Zhang Z, Sun D, Mao X, Liu J, Chen F (2016) The crosstalk between astaxanthin, fatty acids and reactive oxygen species in heterotrophic Chlorella zofingiensis. Algal Res 19:178-183. https://doi.org/10.1016/j.algal.2016.08.015

Zhu L, Li Z, Hiltuen E (2016) Strategies for lipid production improvement in microalgae as a biodiesel feedstock. Biomed Res Int. https:// doi.org/10.1155/2016/8792548 